\title{
Ajuste del modelo Rasch de Escala de Valoración al Cuestionario de Estrés Parental, versión abreviada
}

Andrés Burga

Gracia Sánchez-Griñán

Universidad de Lima

\section{Recibido: 4 de mayo del 2016 / Aprobado: 14 de julio del 2016}

El presente estudio tiene como objetivo determinar si el Cuestionario de Estrés Parental, versión española abreviada, se ajusta al modelo unidimensinal de Rasch. Se ha utilizado un muestreo no probabilístico intencional. La muestra está compuesta por 370 padres de familia de Lima Moderna con hijos entre 0 y 3 años de edad. Los resultados obtenidos señalan que los 23 items que quedan en la versión propuesta muestran un buen ajuste al modelo Rasch de Escala de Valoración. Existe buena confiabilidad de las puntuaciones derivadas de aplicar este instrumento.

estrés parental / cuestionario / modelo Rasch / escala de valoración

\section{Rasch Model Scale Fit Applied to the Parental Stress Index-Short Form (PSI/SF)}

The purpose of this study is to determine whether the Parental Stress Index-Short Form (PSI/SF) fits the unidimensional Rasch model. An intentional non-probabilistic sample composed of 370 parents from metropolitan Lima with children aged 0 to 3 was used. Results indicate that the 23 items in this proposed version show a good fit to the Rasch model. There are good reliability scores derived from the application of this instrument.

parental stress / questionnaire / Rasch model/ rating scale

Correo electrónico: aburga@ulima.edu.pe 


\section{INTRODUCCIÓN}

El estrés es un fenómeno psicofisiológico que se produce en respuesta a diferentes estímulos que se evalúan como inmanejables, y está influenciado por la presencia de distintos factores, sean intrínsecos o extrínsecos a la persona. Puede variar en su intensidad de acuerdo a cómo se percibe y afronta, y tiene efectos distintos en la salud mental y física (Lazarus y Folkman, 1986; Sandín, 2003). Se viene estudiando la implicancia que tiene en diferentes ámbitos como en la escuela, en el trabajo, en la salud, y su relación con las enfermedades (Amigo, 2014; Oblitas, 2008; Rivera de, 2010). Sin embargo, existen otros tipos de situaciones que pueden generar estrés en las personas, por ejemplo, el nacimiento de un nuevo hijo. La paternidad o maternidad es una nueva etapa en la vida que, si bien puede traer mucha felicidad, también trae consigo algunas dificultades.

Durante este segundo periodo del ciclo vital familiar, es natural que surjan distintos pensamientos y sentimientos en los padres y las madres, pues deben integrar a un nuevo ser dentro de la familia y reorganizar sus relaciones, roles y funciones. Asumir un rol paternal o maternal no solo implica una dimensión biológica, sino que también constituye un proceso de cambio psicológico individual y en la pareja (Janto, 2015; Millán, 1996; Santrock, 2006).

\section{ESTRÉS PARENTAL}

El estrés parental puede ser definido como un proceso psicológico en el cual la persona, al ingresar en la etapa de la paternidad, evalúa las exigencias de su rol de padre o madre como algo que excede sus recursos para manejarlo (Lazarus y Folkman, 1986; Sánchez-Griñán, 2015).

$\mathrm{Si}$ bien las personas que ingresan a esta nueva etapa pueden atravesar cambios psíquicos similares, como la constelación maternal (Stern, 1994), la experiencia de cada una es distinta, ya que su personalidad, historia familiar, estilo de vida y contexto en el que viva tendrán un impacto en la manera de asumir esta responsabilidad.

Para poder afrontar el estrés, es necesario que la persona logre encontrar un equilibrio entre las demandas que le exige el ambiente y los recursos con los que cuenta (Lazarus y Folkman, 1986). En el estrés producido por la paternidad o maternidad, las exigencias son muy variadas e implican una adaptación a los atributos del hijo, así como al nuevo rol social de padre o madre. Las demandas más comunes son satisfacer las necesidades del hijo; por ejemplo, alimentación, cuidado, protección, afecto, ayudarlo a regular sus conductas y emociones, etc. En cuanto a los recursos, estos van a depender de muchas variables personales y situacionales como el autoconcepto, los rasgos de personalidad, el sentido de competencia, la situación financiera, el acceso a información y servicios, nivel de educación, soporte social, etc. No obstante, a pesar de que los padres de familia puedan contar con distintos recursos, esto no necesariamente implica que estarán libres de experimentar estrés, pues lo que más importa es la experiencia subjetiva que tienen acerca 
de la paternidad y crianza de los hijos (Cowan y Cowan, 2000; Mossop, 2013; Parker y Hunter, 2011; Valdés, 2007).

A lo largo de la historia, ser padre o madre ha sido percibido como un evento natural que no necesita una preparación formal previa. En consecuencia, muchos padres de familia educan a sus hijos siguiendo su sentido común y sobre la base de los consejos de los familiares, quienes frecuentemente pueden contradecirse, y generar así confusión, frustración, incertidumbre y estrés (Santrock, 2006). Asimismo, las creencias culturales y las expectativas aún tradicionales de la sociedad en el rol materno y paterno (Auad, 2009; Janto, 2015; Marcos, 2010) llevan a los padres y a las madres a cumplir con estas funciones predeterminadas, y a aparentar un estado de plena alegría ante el nacimiento de un nuevo hijo, que los obliga a reprimir su frustración y agotamiento, ya que podrían ser juzgados como "malos" padres si los expresan o si priorizan otros proyectos personales o profesionales (Sánchez-Griñán, 2015).

Dado que el estrés es algo que muchos padres de familia desean ocultar ante los otros, sea por temor o culpa, los profesionales no siempre lo detectan y tratan" (Sánchez-Griñán, 2015). Por ello, es esencial contar con un instrumento psicológico que permita identificar y medir específicamente el estrés parental.

\section{Cuestionario de Estrés Parental}

El Cuestionario de Estrés Parental es un instrumento psicológico que pretende medir el estrés por la crianza de los hijos en los padres de familia. Originalmente, fue elaborado por Abidin en 1983 y revisado con Loyd (1985) en Estados Unidos. Estaba constituído por 120 ítems agrupados en dos dimensiones (dominio del padre y dominio del niño) y presentaba propiedades psicométricas satisfactorias. Luego, fue traducido y adaptado al español por Abidin y Solis (1991), quienes determinaron que tres factores permitían interpretar mejor el estrés parental en la muestra española, y la prueba también presentó buenas propiedades psicométricas en esta población. En consecuencia, los tres factores definidos fueron los siguientes: características del padre, características del niño y la interacción entre padres e hijos, que se ajustaron al modelo multidimensional de estrés por la crianza de Abidin (1995).

Este modelo teórico señala que los factores que generan estrés son aditivos y pueden ser externos, como el temperamento, las necesidades y la adaptabilidad del hijo, así como internos, referidos al sentido de competencia, la personalidad, resiliencia y autoeficacia de los padres al asumir sus responsabilidades en la crianza. De esta manera, la relación que se forma entre ambos puede también ser una fuente de estrés (Abidin, 1995; Abidin y Loyd, 1985; Deater-Deckard, 2004; Pérez y González, 2007).

Es así que el Cuestionario de Estrés Parental está compuesto por tres dimensiones. La primera dimensión se denomina malestar paterno, la cual se refiere a los conflictos con la pareja en los estilos de crianza, la falta de apoyo para cuidar al hijo, el sentido de competencia para atender las necesidades del 
hijo, la percepción que tienen los padres y las madres sobre su rol como tal, etc. interacción disfuncional padre-hijo es la segunda dimensión y aborda la relación conflictiva que surge entre el padre y el hijo. Esta interacción, que se va formando a lo largo del tiempo, se relaciona con las expectativas que tienen los padres de familia acerca del desarrollo de su hijo, la percepción sobre la calidad de la relación entre ambos y el reforzamiento que reciben de sus hijos. La tercera dimensión se denomina niño difícil, y mide el grado en que el estrés parental se ve potenciado por las características propias del hijo, como temperamento, necesidades, regulación de conducta y emociones, estado de ánimo, distractividad, etc.

La prueba original fue transformada a una versión reducida dado el tiempo que requería para su aplicación. De esta manera, la versión abreviada quedó compuesta por 36 ítems en total, agrupados en las tres dimensiones mencionadas. También fue traducida al español y obtuvo propiedades psicométricas satisfactorias, al igual que la versión en inglés (Abidin, 1995; Díaz-Herrero, Brito de la Nuez, López Pina, Pérez-López, y MartínezFuentes, 2010, 2011).

La versión española y abreviada ha sido recientemente adaptada a la población peruana sobre la base de una muestra de 370 padres de familia con hijos e hijas de 0 a 3 años de edad de Lima. En esta nueva versión, también se hallaron las tres dimensiones correlacionadas que permitieron inferir el estrés parental como variable subyacente (Sánchez-Griñán, 2015). En esta versión, se eliminaron
12 ítems, por lo que terminó estando compuesta por 24 ítems en total, ya que algunos no aprobaron el criterio de jueces (Escurra, 1988), y otros saturaban en más de un factor simultáneamente o tenían cargas factoriales menores a .30 (Hair, Anderson, Tatham, y Black, 1999). Se halló evidencias de validez vinculadas a la estructura interna y al contenido (mediante el criterio de jueces), capacidad discriminativa de los ítems y alta confiabilidad para las puntuaciones derivadas de aplicar el instrumento (Sánchez-Griñán, 2015). Finalmente, este instrumento cuenta con baremos generales elaborados sobre la base de la muestra utilizada mediante percentiles.

El presente estudio pretende evaluar la solidez de la interpretación unidimensional de las puntuaciones de este test, en su versión abreviada y española, en la población peruana. Por tanto, se cuestiona si el Cuestionario de Estrés Parental se ajusta al modelo unidimensional de Rasch.

\section{Método}

\section{Participantes}

Se ha utilizado una muestra no probabilística de 370 padres de familia con hijos e hijas que se encontraban entre los 0 y 3 años de edad, y que pertenecían a Lima Moderna (Ipsos APOYO Opinión y Mercado, 2012). Los participantes poseían entre 1 y 4 hijos, uno en la mayoría de casos (57,57 \%). Las edades oscilaban entre los 20 y 50 años, 34 fue el promedio y el $87,84 \%$ pertenecía a la adultez temprana. La mayoría de la muestra estuvo 
compuesta por madres $(88,16 \%)$, mientras que el $17,84 \%$ por padres. Asimismo, el 60,27\% de los participantes recibía apoyo de una tercera persona para cuidar a su hijo, mientras que el $39,73 \%$ no lo tenía.

\section{Instrumento}

El Cuestionario de Estrés Parental, versión abreviada y adaptada a la población española, está compuesto por 36 ítems en total, agrupados en tres dimensiones. Estos son malestar paterno, interacción disfuncional padre-hijo y niño difícil, y cuentan con 12 ítems cada una.

El formato de la prueba es de autoinforme y los ítems corresponden a enunciados que describen situaciones cotidianas de la crianza de los hijos. Los participantes respondieron en una escala Likert de cinco niveles (mиy de acuerdo, de acuerdo, no estoy seguro, desacuerdo, muy en descuerdo). El valor mínimo es 1 cuando el participante marca muy en desacuerdo, y 5 es el máximo al marcar muy de acuerdo. En los ítems 22 y 32, el puntaje máximo es 5 cuando se elige la opción 1, y el mínimo es 1 al elegir la opción 5. Similarmente, en el ítem 33, se otorga 5 puntos cuando el participante marca 10 o más conductas que le molestan de su hijo, y 1 punto cuando indican entre 1 a 3 conductas. Por lo tanto, el puntaje máximo que se puede alcanzar para la prueba total es 180 y el mínimo es 36. Para cada dimensión, el puntaje máximo es 60 y el mínimo es 12. En esta versión española, las puntuaciones que se ubican por encima del percentil 85 y por debajo del percentil
20 tienen significación clínica (Díaz-Herrero et al., 2010).

Con respecto a las propiedades psicométricas de la prueba, los resultados obtenidos sobre la base de una muestra de padres españoles determinaron la existencia de tres factores que, en conjunto, explicaron el 47,48 \% de la varianza común mediante el análisis factorial exploratorio con rotación promax. Además, los ítems presentaron altos coeficientes de consistencia interna para las puntuaciones de la dimensión malestar paterno (.82), Interacción Disfuncional Padre-Hijo (.84), niño difícil (.82), y de la prueba total (.90) (DíazHerrero et al., 2011). Resultados similares con respecto a las propiedades psicométricas se han hallado en estudios realizados en el Perú utilizando esta versión de la prueba (Mejía, 2013; Mendoza, 2014; Pineda, 2012; Sánchez-Griñán, 2015).

\section{Procedimiento}

Se entregó una carta de presentación a los directores de nidos y pediatras con el fin de solicitar autorización y coordinar la aplicación de la prueba a los padres de familia que asistían a sus centros. Los participantes firmaron el consentimiento informado de manera voluntaria y se les garantizó la confidencialidad de sus nombres y de las instituciones. Los padres de familia respondieron de manera individual, y cada aplicación tomó entre 10 y 15 minutos.

\section{Resultados}

Entre los distintos modelos psicométricos que se pueden aplicar a escalas tipo 
Likert, en esta investigación, se aplicó el modelo de Escala de Valoración de Andrich, que considera que una persona, al enfrentarse a una escala tipo Likert, realiza un conjunto de elecciones dicotómicas para llegar a emitir su respuesta (Wright y Masters, 1982). Supongamos que hay un ítem que debe responderse en una escala de cinco puntos: nunca, casi nunca, a veces, siempre y casi siempre. La primera dicotomía podría estar en la selección entre nunca y casi nunca. Si esa persona decide que casi nunca representa sus creencias mejor que nunca, seleccionará esta categoría y la contrastará con la siguiente, y decidirá nuevamente entre una dicotomía, esta vez caracterizada por casi nunca y a veces. De esta manera, si el ítem tiene una escala de respuesta con $j$ categorías, habrá $k=j-1$ umbrales que distingan cada par de categorías consecutivas. Específicamente, el modelo se expresa mediante la siguiente ecuación (Ostini y Nering, 2006):

$$
P\left(X_{i s}=x \mid \theta_{s}, b_{i}, \tau_{k}\right)=\frac{e^{\sum_{k=0}^{x} \theta_{s}-\left(b_{i}-\tau_{k}\right)}}{\sum_{x=0}^{m} e^{\sum_{k=0}^{x} \theta_{s}-\left(b_{i}-\tau_{k}\right)}}
$$

En ella, $m$ representa el valor máximo de la escala de valoración, que empieza en $0 ; \tau_{k}$ es el k-ésimo umbral de la escala de valoración, común a todos los ítems de la prueba y $b_{i}$ representa la localización del ítem en el continuo del rasgo latente $(\theta)$ que se desea medir (Linacre, 2001).

El buen ajuste a un modelo es una parte importante en cualquier análisis estadístico, pero en el caso del análisis Rasch es una parte esencial. Los valores que no se ajustan (misfit) conducen al rechazo de los datos, ya que no cumplen con los requisitos del modelo psicométrico especificado. Como lo señala Wilson (2005), el ajuste de los ítems a un modelo Rasch se basa en dos estadísticos, el outfit e infit, cuyo cálculo se basa en los residuos cuadráticos estandarizados que quedan luego de ajustar un modelo. También se puede calcular la correlación entre la respuesta al ítem y la medida estimada para cada persona $\left(r_{x v}\right)$, que es análoga al índice de discriminación de la teoría clásica de los tests (Linacre, 2005). Se presentan en la Tabla 1 los diferentes indicadores de ajuste calculados para los ítems que conforman el Cuestionario de Estrés Parental, versión peruana abreviada. Además, la Figura 1 muestra las curvas de respuesta correspondientes al modelo de Escala de Valoración de Andrich, estimado para la escala tipo Likert utilizada en el Cuestionario de Estrés Parental. 
Tabla 1

Medida, error e indicadores de ajuste de los ítems de la prueba.

\begin{tabular}{|c|c|c|c|c|c|}
\hline Ítem & medida & error & infit & outfit & rim \\
\hline MP1 & -0.913 & 0.058 & 1.01 & 1.17 & .56 \\
\hline MP2 & -1.481 & 0.056 & 1.14 & 1.15 & .59 \\
\hline MP3 & -0.923 & 0.058 & 1.21 & 1.19 & .54 \\
\hline MP5 & -1.052 & 0.057 & 1.13 & 1.13 & .57 \\
\hline MP12 & -0.220 & 0.066 & 1.29 & 1.19 & .53 \\
\hline IDPH13 & 0.312 & 0.078 & 1.02 & 0.84 & .55 \\
\hline IDPH14 & 1.526 & 0.124 & 1.17 & 0.83 & .37 \\
\hline IDPH16 & 0.904 & 0.096 & 1.10 & 0.80 & .48 \\
\hline IDPH19 & 1.194 & 0.108 & 1.21 & 1.18 & .34 \\
\hline IDPH20 & 1.062 & 0.102 & 1.18 & 0.93 & .41 \\
\hline IDPH21 & 0.191 & 0.075 & 1.28 & 1.25 & .43 \\
\hline IDPH23 & 0.520 & 0.084 & 1.21 & 0.99 & .47 \\
\hline IDPH24 & 0.412 & 0.080 & 0.99 & 0.76 & .56 \\
\hline ND25 & 0.174 & 0.074 & 1.07 & 0.94 & .54 \\
\hline ND26 & 0.147 & 0.074 & 0.98 & 1.02 & .49 \\
\hline ND27 & -0.497 & 0.062 & 0.93 & 0.85 & .63 \\
\hline ND28 & 0.052 & 0.071 & 0.79 & 0.72 & .61 \\
\hline ND29 & -0.967 & 0.058 & 1.15 & 1.13 & .59 \\
\hline ND30 & -0.355 & 0.064 & 0.87 & 0.80 & .63 \\
\hline ND32 & -1.538 & 0.056 & 0.99 & 1.21 & .53 \\
\hline ND34 & 0.141 & 0.073 & 0.88 & 0.78 & .58 \\
\hline ND35 & 1.160 & 0.106 & 0.86 & 0.59 & .52 \\
\hline ND36 & 0.152 & 0.074 & 1.22 & 1.21 & .51 \\
\hline
\end{tabular}




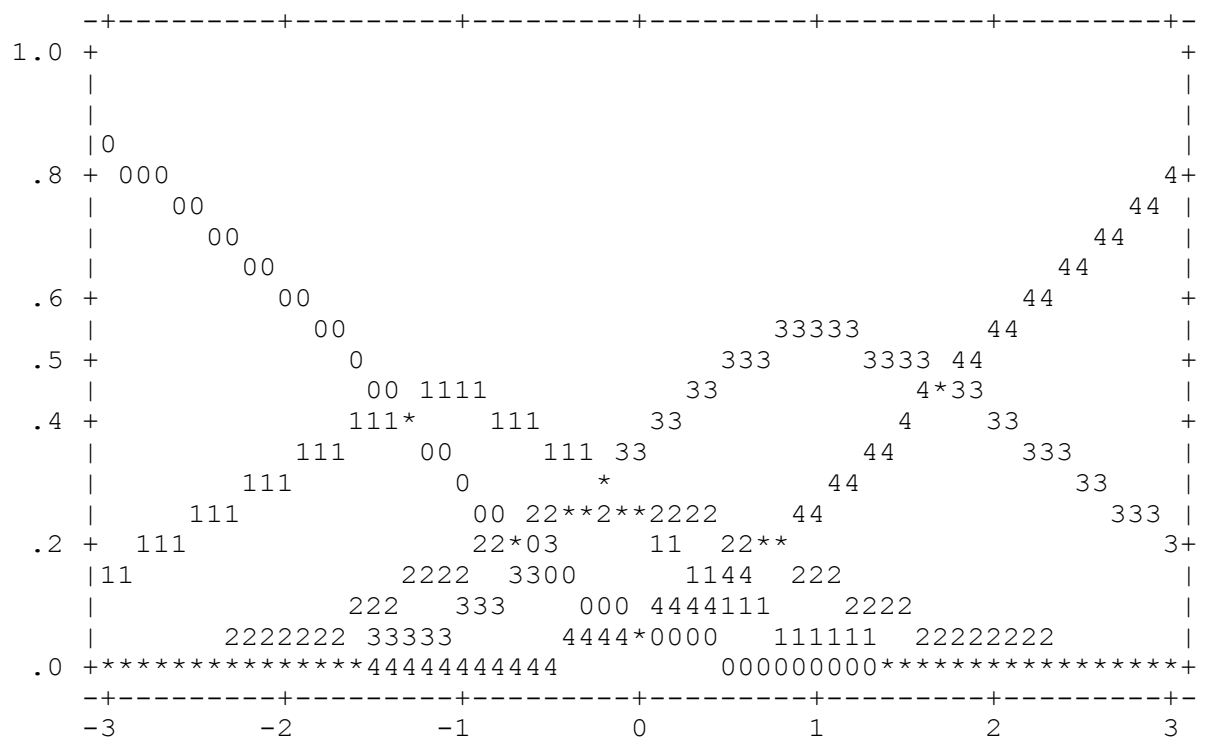

Figura 1. Curvas de probabilidad de las categorías para la escala de valoración de cinco puntos utilizada

La confiabilidad de las medidas derivadas de aplicar el Cuestionario de Estrés Parental en este grupo de personas se estimó mediante el Indice de Confiabilidad de la Separación de Personas (Rp). Su cálculo se basa en la información referida a la variabilidad contenida en una sola aplicación del instrumento; concretamente, se refiere a la proporción de varianza explicada por el estimador de la medida de habilidad de una persona (Wilson, 2005). Este coeficiente sirve para indicar la capacidad de las medidas de un test para diferenciar las cantidades de rasgo latente que poseen los evaluados (Wrigth y Masters, 1982). Además, se calculó el índice de separación de personas (Gp), que se refiere a la dispersión de los datos medidos como el número de errores estándar que separan a las personas (Schumacker, 2007). La Tabla 2 muestra los valores de ambos índices, estimados en la muestra objeto de estudio.

Tabla 2

Evidencias de confiabilidad de las medidas derivadas de la aplicación de la prueba

\begin{tabular}{lc}
\hline Coeficiente & Valor \\
\hline $\mathrm{Rp}$ & .88 \\
$\mathrm{Gp}$ & 2.75 \\
\hline
\end{tabular}


Las evidencias de unidimensionalidad desde los modelos Rasch se obtienen al realizar un análisis de componentes principales de los residuos estandarizados luego de ajustar el modelo Rasch unidimensional (Linacre, 2005). El análisis factorial de los residuos sirve para detectar cualquier varianza que queda en los datos luego de extraer la dimensión principal (Bond y Fox, 2015). Los resultados de dicho análisis pueden ser consultados en la Tabla 3.

Tabla 3

Evidencias de unidimensionalidad

\begin{tabular}{l|c}
\hline \multicolumn{1}{c|}{ Indicador } & Valor \\
\hline $\begin{array}{l}\text { Varianza de la dimensión } \\
\text { principal (\%) }\end{array}$ & 66.8 \\
\hline Varianza modelada (\%) & 51.2 \\
\hline Primer autovalor & 3.3 \\
\hline$\%$ del primer autovalor & 7.4 \\
\hline
\end{tabular}

\section{DISCUSIÓN}

El objetivo de esta investigación fue analizar la estructura unidimensional del Cuestionario de Estrés Parental, versión española abreviada, en una muestra de 370 padres de familia con hijos e hijas entre 0 a 3 años de edad que pertenecen a los distritos de Lima Moderna (Ipsos APOYO Opinión y Mercado, 2012). Para ello, se aplicó el modelo Rasch de Escala de Valoración de Andrich. Los resultados del análisis del ajuste de ítems se obtuvieron siguiendo las recomendaciones de
Linacre (2005), quien señala que se espera que los ítems con un ajuste adecuado al modelo Rasch tengan valores infit y outfit entre 0.50 y 1.50 , y se prefieren los valores entre 0.70 y 1.30 (Wright y Linacre, 1994). Es importante señalar que algunos autores (Schulz, 1990) consideran que debe prestársele mayor atención al infit, ya que es menos sensible a las variaciones en el tamaño de la muestra utilizada para calibrar los ítems. Además, se esperan correlaciones ítem-medida (ptme) positivas (Linacre 2005).

Como se pudo observar en la Tabla 1 , se eliminaron los ítems $4,8,11,15,18,22$, 31 y 33 por no mostrar un adecuado ajuste al modelo Rasch, según los parámetros antes señalados. Coincidentemente, los ítems 22 y 31 fueron también eliminados en el estudio de Sánchez-Griñán (2015), ya que presentaban una carga menor a .30 y saturaban en más de un factor. Es posible que, en esta muestra de personas, los ítems no estén midiendo con suficiente fuerza la dimensión principal que pretende explicar las covariaciones observadas entre los ítems, es decir, el estrés parental. En el resto de ítems, los indicadores de ajuste son buenos. Por lo tanto, pueden ser considerados en la estimación de la medida del rasgo latente de las personas evaluadas, pues funcionan de acuerdo con lo esperado por el modelo Rasch utilizado. Se ha podido observar, además, que no todas las categorías de la Escala de Valoración han resultado ser valores modales, lo que produce umbrales Rasch-Andrich desordenados; estos fueron los valores observados: $-1.35,-0.09,-0.29,1.72$. 
Sobre los valores mínimos aceptables de los coeficientes de confiabilidad, Charter (2003) ha realizado una revisión de numerosas investigaciones en las que se proponen diferentes niveles mínimos. En ese estudio, se encontró bastante variabilidad observando valores, propuestos con los diversos métodos para obtener la confiabilidad, que oscilan entre .60 y .95 . A pesar de esta gran variabilidad, un estándar mínimo aceptable que aparece con frecuencia en la literatura es el de .70. Por ende, el análisis de la confiabilidad de las medidas derivadas de aplicar el Cuestionario de Estrés Parental no señala ningún problema en este aspecto.

Considerando el análisis de la unidimensionalidad de las medidas, Linacre (2005) señala que, si bien no existen parámetros absolutos para interpretar los resultados del análisis de componentes principales de los residuos, si el primer autovalor es menor que $3.0 \mathrm{y} / \mathrm{o}$ contiene menos del $5 \%$ de varianza, no hay serias evidencias que atenten contra el supuesto de unidimensionalidad. Además, este mismo autor señala que se está frente a datos unidimensionales cuando la cantidad de varianza empírica es similar a la cantidad de varianza predicha por el modelo. Si bien el porcentaje de varianza explicada por el primer autovalor es algo elevado, en el mejor de los casos, la dimensión secundaria está conformada solo por 3 ítems, todos ellos pertenecientes a malestar paterno. Esto se podría deber a que el estrés parental se ve más influenciado por las características propias de los hijos (niño difícil), pues cada uno de ellos presenta rasgos únicos y distintos que afectan la manera en que los padres de familia perciben las demandas de la crianza, y por lo tanto se ve afectada la relación conflictiva que puede surgir (interacción disfuncional padre-hijo). De esta manera, es posible que Malestar Paterno no ejerza tanto impacto en el estrés como las otras dos dimensiones, pues las características personales de los padres tienden a mantenerse en el tiempo y a ser las mismas más allá de los atributos psicológicos de los niños. Pareciera que las conductas y el temperamento de los niños, y por ende la interacción entre padres e hijos, ejercen mayor impacto en el estrés parental que las características personales de los padres.

A pesar de ello, se puede considerar que, en general, estamos frente a un conjunto de ítems que no presenta serias desviaciones del supuesto de unidimensionalidad propio de los modelos Rasch.

A manera de conclusión, se puede señalar que los 23 ítems que quedan en esta versión propuesta del Cuestionario de Estrés Parental muestran un buen ajuste al modelo Rasch de Escala de Valoración. La confiabilidad de las medidas derivadas de aplicar este instrumento es buena, y no muestran serias evidencias en contra del supuesto de unidimensionalidad. Por lo tanto, se recomienda aplicarlo y calificarlo desde el modelo Rasch unidimensional utilizado en esta investigación. 


\section{ReFERENCIAS}

Abidin, R. R. (1995). Parenting Stress Index-Short Form (PSI-SF): Professional Manual (3. a ed.). Odessa, FL: Psychological Assessment Resources. Recuperado del sitio de internet Psychological Assessment Resources: http://www4.parinc.com/

Abidin, R. R, y Loyd, B. (1985). Revision of Parenting Stress Index. Journal of Pediatric Psychology, 10(2), 169-177. doi:10.1093/jpepsy/10.2.169

Abidin, R. R, y Solis, M. L. (1991). The Spanish Version Parenting Stress Index: A Psychometric Study. Journal of Clinical Child Psychology, 20(4), 372378. doi:10.1207/s15374424jccp2004_5

Amigo, I. (2014). Manual de psicología de la salud (2. ${ }^{\mathrm{a}}$ ed.). Madrid: Ediciones Pirámide.

Auad, M. (2009). Representaciones mentales de la maternidad en madres de hijos con trastorno del espectro autista (Tesis de licenciatura). Recuperado de http://tesis.pucp.edu.pe/

Bond, T. G., y Fox, C. M. (2015). Applying the Rasch Model. Fundamental Measurement in the Human Sciences (3. ${ }^{\mathrm{a}}$ ed.). New York, NY: Routledge.

Charter, R. A. (2003). A Breakdown of Reliability Coefficients by Test Type and Reliability Method, and the Clinical Implications of Low Reliability. The Journal of General Psychology, 130(3), 290-304.

Cowan, C. A., y Cowan, P. A. (2000). When Partners Become Parents: the Big Life
Change for Couples (2. ${ }^{\mathrm{a}}$ ed.). Mahwah, NJ: Lawrence Erlbaum Associates.

Deater-Deackard, K. (2004). Parenting Stress. New Heaven, CT: Yale University Press.

Díaz-Herrero, Á., Brito de la Nuez, A., López Pina, J., Pérez-López, J., y Martínez-Fuentes, M. (2010). Estructura factorial y consistencia interna de la versión española del Parenting Stress Index-Short Form. Psicothema, 22(4), 1033-1038. Recuperado de http:// www.psicothema.com/pdf/3837.pdf

Díaz-Herrero, Á., Brito de la Nuez, A., López Pina, J., Pérez-López, J., y Martínez-Fuentes, M. (2011). Validity of the Parenting Stress Index-Short Form in a Sample of Spanish Fathers. The Spanish Journal of Psychology, 14(2), 990-997. Recuperado de http:// dx.doi.org/10.5209/rev_SJOP.2011. v14.n2.44

Escurra, L. M. (1998). Cuantificación de la validez de contenido por criterio de jueces. Revista de Psicología, 6(2), 103-111. Recuperado de http://revistas.pucp.edu.pe/

Everitt, B. S., y Skrondal, A. (2010). The Cambridge dictionary of statistics $\left(4 .^{\mathrm{a}}\right.$ ed.). New York, NY: Cambridge University Press.

Hair, J., Anderson, R., Tatham, R., y Black, W. (1999). Análisis multivariante (5. ${ }^{\text {a }}$ ed.). Madrid: Prentice Hall.

Ipsos APOYO Opinión y Mercado. (2012). Perfiles zonales de la Gran Lima. Recuperado de http://www.ipsos.pe/sites/ 
default/files/marketing_data/Marketing\%20data\%20PZ\%202012.pdf

Janto, C. (2015). La paternidad: una creación compartida (Tesis de licenciatura). Recuperado de http://tesis. pucp.edu.pe/

Lazarus, R., y Folkman, S. (1986). Estrés y procesos cognitivos. Madrid: Martínez Roca.

Linacre, J. M. (2005). Winsteps Manual. Chicago: MESA.

Marcos, C. (2010). Representaciones mentales de la paternidad en padres varones adolescentes (Tesis de licenciatura). Recuperado de http://tesis. pucp.edu.pe/

Mejía, M. (2013). Estresores relacionados con el cáncer, sentido de coherencia y estrés parental en madres de niños con leucemia que provienen del interior del país (Tesis de maestría). Recuperada de http://tesis.pucp.edu.pe/

Mendoza, X. (2014). Estrés parental y optimismo en padres de niños con trastorno del espectro autista (Tesis de licenciatura). Recuperado de http:// tesis.pucp.edu.pe/

Millán, M. (1996). Psicología de la familia: un enfoque evolutivo y sistémico (vol. 1). Valencia: Promolibro.

Mossop, B. (2013). Cerebro y paternidad. Mente y Cerebro, 58, 28-33.

Oblitas, L. (2008). El estado del arte de la psicología de la salud. Revista de Psicología, 26(2), 222-251. Recuperado de http://revistas.pucp.edu.pe/
Ostini, R., y Nering, M.L. (2006). Polytomous Item Response Theory Models. Quantitative Applications for the Social Sciences 144. Thousand Oaks, CA: Sage Publications.

Parker, R., y Hunter, C. (2011). Supporting Couples Across the Transition to Parenthood (Reporte n. 20 2011). Recuperado del sitio de internet Australian Institute of Family Studies: https://www3.aifs. gov.au/cfca/sites/default/files/publication-documents/bp020.pdf

Pérez, G., y González, M. (2007). Crianza y desarrollo infantil en familia yoreme-mayo en el norte de sinaloa (Tesis de licenciatura, Universidad Autónoma Indígena de México, Mochicahui). Recuperado de http:// www.ciad.mx/archivos/desarrollo/ publicaciones/Tesis\%20asesoradas/ Licenciatura/42.pdf

Pineda, D. (2012). Estrés parental y estilos de afrontamiento en padres de niños con trastornos del espectro autista (Tesis de licenciatura). Recuperado de http://tesis.pucp.edu.pe/

Rivera de, L. (2010). Los síndromes de estrés. Madrid: Síntesis

Sánchez-Griñán, G. (2015). Cuestionario de estrés parental: características psicométricas y análisis comparativo del estrés parental en padres de familia con hijos e hijas de 0 a 3 años de edad de Lima Moderna (Tesis de licenciatura inédita). Universidad de Lima, Perú.

Sandín, B. (2003). El estrés: un análisis basado en el papel de los factores 
sociales. Revista Internacional de Psicología Clínica y de la Salud, 3(1), 141-157. Recuperado de http://www. redalyc.org/articulo.oa?id=33730109

Santrock, J. (2006). Psicología del desarrollo: el ciclo vital (10. ${ }^{\mathrm{a}}$ ed.). Madrid: McGraw Hill Interamericana.

Schulz, M. (1990). Functional Assessment of Fit. En J. M. Linacre (Ed.), Rasch Measurement Transactions Part I (pp. 82-84). Chicago: MESA Press.

Schumacker, R. (2007). Reliability: a Rasch perspective. Educational and Psychological Measurement, 67(3), 394-409.

Stern, D. (1994). La constelación maternal. Barcelona: Paidós.

Wilson, M. (2005). Constructing measures. An item response modeling approach. Mahwah, NJ: Lawrence Erlbaum Associates.

Wright, B. D., y Linacre, J. M. (1994). Reasonable Mean - Square Fit Values. En J. M. Linacre (Ed.), Rasch Measurement Transactions Part II (p. 370). Chicago: MESA Press.

Wright, B. D., y Masters, G. N. (1982). Rating Scale Analysis. Chicago: MESA.

\section{ANEXO}

Sintaxis de Winsteps utilizada para ajustar el modelo Rasch

\&INST

TITLE $=$ CEP

DATA $=$ CEP.dat

$$
\begin{aligned}
& \text { PERSON }=\text { Alumno } \\
& \text { ITEM }=\text { Item } \\
& \text { ITEM } 1=5 \\
& \text { NI }=36 \\
& \text { NAME } 1=1 \\
& \text { XWIDE }=1 \\
& \text { LCONV }=.001 \\
& \text { MISSCORE }=-1 \\
& \text { CODES }=01234 \\
& \text { NORMAL }=\text { N } \\
& \text { LOCAL }=\text { Y } \\
& \text { PTBIS }=\text { N } \\
& \text { DISCRIM }=\text { YES } \\
& \text { PVALUE }=\text { YES } \\
& \text { ASYMPTOTE }=\text { YES }
\end{aligned}
$$

\section{IDFILE $=*$}

6; eliminado por criterio de jueces

7; eliminado por criterio de jueces

9 eliminado por criterio de jueces 10; eliminado por criterio de jueces 17; eliminado por criterio de jueces 15 
22

*

USCALE $=1$

UDECIM $=3$

MODEL $=\mathrm{R}$

\&END

MP1

MP2

MP3

MP4

MP5

MP6

MP7

MP8

MP9

MP10

MP11

MP12

IDPH13

IDPH14

IDPH15

IDPH16
IDPH17

IDPH18

IDPH19

IDPH20

IDPH21

IDPH22

IDPH23

IDPH24

ND25

ND26

ND27

ND28

ND29

ND30

ND31

ND32

ND33

ND34

ND35

ND36

END LABELS 